\title{
Contractivity and global stability for discrete models of Lotka-Volterra type
}

\author{
Yoshiaki MuroyA
}

(Received April 2, 2003; Revised February 16, 2004)

Abstract. Consider the following discrete model of nonautonomous Lotka-Volterra type:

$$
\left\{\begin{array}{r}
N_{i}(p+1)=N_{i}(p) \exp \left\{r_{i}(p)\left(c_{i}(p)-b_{i}(p) N_{i}(p)-\sum_{j=1}^{n} \sum_{l=0}^{m} b_{i j}^{l}(p) N_{j}(p-l)\right)\right\} \\
p=0,1,2, \ldots, \quad 1 \leq i \leq n \\
N_{i}(0)=N_{i 0}>0 \quad \text { and } \quad N_{i}(-l)=N_{i(-l)} \geq 0, \quad 1 \leq i \leq n, 1 \leq l \leq m
\end{array}\right.
$$

where

$$
\left\{\begin{array}{l}
r_{i}(p)>0, \quad \text { for } \quad p \geq 0, \liminf _{p \rightarrow \infty} r_{i}(p)>0 \\
b_{i}(p)>0, b_{i i}^{0}(p)=0,1 \leq i \leq n, \quad b_{i j}^{l}(p) \geq 0,1 \leq i \leq j \leq n, 0 \leq l \leq m .
\end{array}\right.
$$

In this paper, to the above discrete system, we establish two type sufficient conditions that ensure the "contractivity" of solutions which are sufficient conditions of the global asymptotic stability of system. This is an extension of the former work (2002, J. Math. Anal. Appl. 270, 602-635) for $n=1$ to $n \geq 2$. In particular, for autonomous case of the above system, the sufficient condition for the global asymptotic stability of the positive equilibrium offered by Wang et al. (2002, J. Math. Anal. Appl. 264, 147-169), is proved to satisfy this contractivity of the positive equilibrium.

Key words: contractivity; global asymptotic stability; discrete model of Lotka-Volterra type.

\section{Introduction}

In this paper, we consider the following discrete model of nonautonomous Lotka-Volterra type:

2000 Mathematics Subject Classification : 34K20, 34K60.

Research partially supported by Waseda University Grant for Special Research Projects 2003A-573. 


$$
\left\{\begin{array}{r}
N_{i}(p+1)=N_{i}(p) \exp \left\{r _ { i } ( p ) \left(c_{i}(p)-b_{i}(p) N_{i}(p)\right.\right. \\
\left.\left.-\sum_{j=1}^{n} \sum_{l=0}^{m} b_{i j}^{l}(p) N_{j}(p-l)\right)\right\}, \\
1 \leq i \leq n, p \geq 0, \\
N_{i}(0)=N_{i 0}>0 \quad \text { and } \quad \begin{array}{r}
N_{i}(-l)=N_{i(-l)} \geq 0, \\
1 \leq i \leq n, 1 \leq l \leq m,
\end{array}
\end{array}\right.
$$

where

$$
\left\{\begin{aligned}
r_{i}(p)>0 \quad \text { for } \quad p \geq 0, \liminf _{p \rightarrow \infty} & r_{i}(p)>0 \\
b_{i}(p)>0, & b_{i i}^{0}(p)=0,1 \leq i \leq n, b_{i j}^{l}(p) \geq 0, \\
& \quad 1 \leq i \leq j \leq n, 0 \leq l \leq m .
\end{aligned}\right.
$$

For autonomous cases in the above discrete population models, there are several literatures. Hofbauer, Hutson and Jansen [3] have shown that the existence of positive equilibrium in the system guarantee its permanence for the case of $n=2$ and $m=0$ that the system is a prey-predator system or the two species are competitive. But if the system is cooperative, $\mathrm{Lu}$ and Wang [4] showed that it can not be permanent in any case. Lu and Wang [4] gave sufficient conditions for permarence for no delay case $m=0$, and later, Saito, Ma and Hara [10] and Saito, Hara and Ma [9] generalized them and for the special system that $n=2$ and any $m \geq 0$, but for each $j$, there is only one $j_{i}$ such that

$$
b_{i j}^{j_{i}} \neq 0, \quad \text { and } \quad b_{i j}^{k}=0, \quad \text { for } \quad k \neq j_{i}, 1 \leq i, j \leq 2,
$$

established necessary and sufficient conditions for permanence.

For $n=1$ and $m \geq 1$, Muroya [5, 7] established sufficient conditions of "contractivity" (see Eq. (1.3)) for solutions of the positive equilibrium of system (1.1).

On the other hand, for nonautonomous cases of $n \geq 2$ under the assumption that the system is persistent, Wang and $\mathrm{Lu}$ [12] for $m=0$ and Wang et al. [13] for $m \geq 0$, established sufficient conditions that ensure that the discrete system is globally asymptotically stable, and in the case of prey-predator and competitive system for $n=2$, they gave sufficient conditions that the system is persistent. For the cases $n \geq 2$ and $m \geq 0$, Muroya [6] established sufficient conditions for the persistence and global 
asymptotic stability of system (1.1) which improved the results of Wang et al. [13]. Muroya [8] offered sufficient conditions for the permanence of system (1.1).

For a discrete model of Lotka-Volterra competitive system for two species, using some kind of contractivity conditions, Chen and Zhou [2] obtained another type sufficient condition for global asymptotic stability of a positive periodic solution for a discrete periodic Lotka-Volterra competitive system (see Eq. (2.38)).

If for a solution $\left\{N_{i}^{*}(p)\right\}_{i=1}^{n}$ of system (1.1), there exists a constant $0 \leq$ $k \leq 1$ such that for any solution $\left\{N_{i}(p)\right\}_{i=1}^{n}$ of system (1.1) and a sufficiently large $p \geq 0$,

$$
\begin{aligned}
& \max _{1 \leq i \leq n}\left|N_{i}(p+1)-N_{i}^{*}(p+1)\right| \\
& \quad \leq k \max \left\{\left|N_{j}(p-l)-N_{j}^{*}(p-l)\right| \mid 1 \leq j \leq n, 0 \leq l \leq m\right\},
\end{aligned}
$$

then we refer that for a sufficiently large $p \geq 0$, solutions of system (1.1) hold the contractivity to the solution $\left\{N_{i}^{*}(p)\right\}_{i=1}^{n}$.

In particular, if $k<1$, then we say that for a sufficiently large $p \geq 0$, solutions of system (1.1) hold the strong contractivity to the solution $\left\{N_{i}^{*}(p)\right\}_{i=1}^{n}$. Note that the contractivity implies that the system is persistent and any solution of system is uniformly stable. Moreover, the strong contractivity implies that any solution of system is globally attractive, and hence is globally asymptotically stable.

In this paper, for the above discrete system, we establish sufficient conditions that ensures the strong contractivity to some solution $\left\{N_{i}^{*}(p)\right\}_{i=1}^{n}$ and hence global asymptotic stability of the system (see Theorem 1.1). In particular, for autonomous case in (1.1), we show that for the condition offered by Wang et al. [13], solutions of system (1.1) hold the strong contractivity to the positive equilibrium of (1.1) (see Theorem 2.1). Moreover, we obtain an extended result of Muroya [5] for $n=1$ to $n \geq 2$ (see Theorem 1.2) and Chen and Zhou [2] for $n=2$ and $m=0$ to $n \geq 2$ and $m \geq 0$ (see Theorem 2.2).

For a given sequence $\{g(p)\}_{p=0}^{\infty}$, we set

$$
\left\{\begin{array}{l}
g_{M}=\sup \{g(p) \mid p=0,1,2, \ldots\} \\
g_{L}=\inf \{g(p) \mid p=0,1,2, \ldots\} .
\end{array}\right.
$$

Put 


$$
\left\{\begin{array}{l}
\tilde{A}_{L}=\operatorname{diag}\left(b_{1 L}, b_{2 L}, \ldots, b_{n L}\right), \tilde{B}_{L}^{-}=\left[\tilde{b}_{i j L}^{-}\right], \hat{\tilde{B}}_{M}=\left[\hat{\tilde{b}}_{i j M}\right], \text { and } \\
\overline{\boldsymbol{R}}=\operatorname{diag}\left(\bar{r}_{1}, \bar{r}_{2}, \ldots, \bar{r}_{n}\right) \text { are } n \times n \text { matrices } \\
\text { with } b_{i j}^{l+}(p)=\max \left(b_{i j}^{l}(p), 0\right) b_{i j}^{l-}(p)=\min \left(b_{i j}^{l}(p), 0\right), \\
\tilde{b}_{i j}^{+}(p)=\sum_{l=0}^{m} b_{i j}^{l+}(p), \tilde{b}_{i j}^{-}(p)=\sum_{l=0}^{m} b_{i j}^{l-}(p), \hat{\tilde{b}}_{i j}(p)=\sum_{l=0}^{m}\left|b_{i j}^{l}(p)\right|, \\
\left.\quad \text { and } \quad \underline{r}_{i}=\inf _{p \geq 0} r_{i}(p), 1 \leq i \leq n\right), \\
\bar{r}_{i}=\sup _{p \geq 0} r_{i}(p), \quad 1 \leq n \geq 0,
\end{array}\right.
$$

and

$$
\left\{\begin{array}{l}
\tilde{f}(t ; r)= \begin{cases}\left(e^{r t}-1\right) / t, & t \neq 0, \\
r, & t=0,\end{cases} \\
f(t ; r)=(1-t) \tilde{f}(t ; r) .
\end{array}\right.
$$

For two solutions $\left\{N_{i}(p)\right\}_{i=1}^{n}$ and $\left\{N_{i}^{*}(p)\right\}_{i=1}^{n}$ of system (1.1), put

$$
\left\{\begin{array}{l}
t_{i}(p)=c_{i}(p)-b_{i}(p) N_{i}(p)-\sum_{j=1}^{n} \sum_{l=0}^{m} b_{i j}^{l}(p) N_{j}(p-l), \\
t_{i}^{*}(p)=c_{i}(p)-b_{i}(p) N_{i}^{*}(p)-\sum_{j=1}^{n} \sum_{l=0}^{m} b_{i j}^{l}(p) N_{j}^{*}(p-l), \\
k_{i}(p)=N_{i}(p) \tilde{f}\left(t_{i}(p)-t_{i}^{*}(p) ; r_{i}(p)\right), \quad 1 \leq i \leq n, p \geq 0 .
\end{array}\right.
$$

We shall establish the following results to the system (1.1).

Theorem 1.1 Assume that there exists a solution $\left\{N^{*}(p)\right\}_{i=1}^{n}$ of (1.1) and a positive constant $k \leq 1$ such that for $1 \leq i \leq n$,

$$
\left\{\begin{array}{l}
\bar{c}_{i M}>0, \bar{r}_{i} b_{i M} \bar{N}_{i} \leq 1, \bar{r}_{i}\left(c_{i M}-\sum_{j=1}^{i-1} \tilde{b}_{i j L}^{-} \bar{N}_{j}-\inf _{p \geq 0} t_{i}^{*}(p)\right) \leq 1, \\
\text { and } \\
\underline{N}_{i} \tilde{f}\left(q_{i} ; \underline{r}_{i}\right) \geq\left\{1-k \exp \left(-\bar{r}_{i} t_{i}^{*}(p)\right)\right\} /\left(b_{i}(p)-\sum_{j=1}^{n} \hat{\tilde{b}}_{i j}(p)\right),
\end{array}\right.
$$

where for $i=1,2, \ldots, n$, 


$$
\left\{\begin{array}{l}
\bar{c}_{i M}=c_{i M}-\sum_{j=1}^{i-1} \tilde{b}_{i j L}^{-} \bar{N}_{j}, \bar{N}_{i}=\frac{\bar{c}_{i M}}{b_{i L}} \\
\tilde{b}_{i M}=b_{i M}+\sum_{l=1}^{m} b_{i i M}^{l} \exp \left\{-k_{l} \bar{r}_{i}\left(c_{i L}-b_{i M} \bar{N}_{i}\right.\right. \\
\left.\left.-\sum_{j=1}^{i-1} b_{i j M}^{-} \underline{N}_{j}-\sum_{j=1}^{n} b_{i j M}^{+} \bar{N}_{j}\right)\right\} \\
\underline{\tilde{N}}_{i}=\left(c_{i L}-\sum_{j=1}^{i-1} b_{i j M}^{-} \underline{N}_{j}-\sum_{j \neq i} b_{i j M}^{+} \bar{N}_{j}\right) / \tilde{b}_{i M} \\
\underline{N}_{i}=\min \left(\underline{\tilde{N}}_{i}, \bar{N}_{i} \exp \left\{\overline { r } _ { i } \left(c_{i L}-\sum_{j=1}^{i-1} b_{i j M}^{-} \underline{N}_{j}\right.\right.\right. \\
\left.\left.\left.-\sum_{j \neq i} b_{i j M}^{+} \bar{N}_{j}-\tilde{b}_{i M} \bar{N}_{i}\right)\right\}\right)>0 \\
q_{i}=\min \left\{c_{i L}-b_{i M} \bar{N}_{i}-\sum_{j=1}^{n} b_{i j}^{+} \bar{N}_{j}-\sup _{p \geq 0} t_{i}^{*}(p), 0\right\}
\end{array}\right.
$$

and

$$
\tilde{A}_{L}-\hat{\tilde{B}}_{M} \text { is an } M \text { matrix. }
$$

Then, for a sufficiently large $p \geq 0$, solutions of system (1.1) hold the contractivity to the solution $\left\{N_{i}^{*}(p)\right\}_{i=1}^{n}$.

Moreover, if $k<1$, then for a sufficiently large $p \geq 0$, solutions of system (1.1) hold the strong contractivity and

$$
\lim _{p \rightarrow \infty}\left(N_{i}(p)-N_{i}^{*}(p)\right)=0, \quad 1 \leq i \leq n .
$$

For nonautonomous case of (1.1), an example is given (see Example 2.1).

If each $c_{i}(p), b_{i}(p)$ and $b_{i j}^{l}(p)$ in (1.1) are constants and there exists a unique positive equilibrium $\boldsymbol{N}^{*}=\left(N_{1}^{*}, N_{2}^{*}, \ldots, N_{n}^{*}\right)^{T}$ of (1.1), then for $\left\{N_{i}^{*}(p)\right\}_{i=1}^{n}=\left\{N_{i}^{*}\right\}_{i=1}^{n}, t_{i}^{*}(p)=0$ and the condition (1.8) becomes a simpler condition as follows.

Theorem 1.2 Assume that each $c_{i}(p), b_{i}(p)$ and $b_{i j}^{l}(p)$ in (1.1) are constants, that is, $c_{i}(p)=c_{i}, b_{i}(p)=b_{i}$ and $b_{i j}^{l}(p)=b_{i j}^{l}$, and there exists the 
positive equilibrium $\boldsymbol{N}^{*}=\left(N_{1}^{*}, N_{2}^{*}, \ldots, N_{n}^{*}\right)^{T}$ of (1.1) and suppose that

$$
\begin{aligned}
c_{i}-\sum_{j=1}^{i-1} \tilde{b}_{i j}^{-} \tilde{N}_{j}>0, \quad \text { and } \quad \bar{r}_{i}\left(c_{i}-\sum_{j=1}^{i-1} \tilde{b}_{i j}^{-} \tilde{N}_{j}\right) \leq 1, & \\
& 1 \leq i \leq n,
\end{aligned}
$$

and

$$
\tilde{A}_{L}-\hat{\tilde{B}}_{M} \text { is an } M \text { matrix. }
$$

Then, for a sufficiently large $p \geq 0$, solutions of system (1.1) hold the strong contractivity and the positive equilibrium $\boldsymbol{N}^{*}=\left(N_{1}^{*}, N_{2}^{*}, \ldots, N_{n}^{*}\right)^{T}$ of system (1.1) is globally asymptotically stable.

Theorem 1.2 is an extension of Muroya [7, Theorem 1.1] for $n=1$ to $n \geq 1$.

Under the strong persistence of system (1.1) (that is, $\liminf _{p \rightarrow \infty} N_{i}(p)>$ $0,1 \leq i \leq n)$, Wang et al. [13] proved that system (1.1) is globally asymptotically stable, and for autonomous case, the condition becomes Eq. (1.12), but nothing says about the strong contractivity, because the proof depends on Lyapunov-like function method (see also Muroya [6]).

Let $\hat{r}(\alpha)$ be a strictly monotone increasing function of $\alpha$ on the interval $(-1,1)$ such that there exists $\hat{t}(\alpha)<1$ and

$$
\left\{\begin{array}{l}
f(\hat{t}(\alpha) ; \hat{r}(\alpha))=2 /(1-\alpha), \\
f^{\prime}(\hat{t}(\alpha) ; \hat{r}(\alpha))=0
\end{array}\right.
$$

The following theorem is an extension of Muroya [7, Theorem 2.1] for $n=1$ to the system (1.1) for $n \geq 1$.

Theorem 1.3 Assume that each $c_{i}(p), b_{i}(p)$ and $b_{i j}^{l}(p)$ in (1.1) are constatnts, that is, $c_{i}(p)=c_{i}, b_{i}(p)=b_{i}, b_{i j}^{l}(p)=b_{i j}^{l}$, and

$$
\left\{\begin{array}{l}
b_{i}>\sum_{j=1}^{n} \hat{\tilde{b}}_{i j}, \quad \text { and } \\
\bar{r}_{i} \leq \hat{r}\left(-\left(\sum_{j=1}^{n} \hat{\tilde{b}}_{i j}\right) / b_{i}\right) /\left(c_{i}-\sum_{j=1}^{i-1} \tilde{b}_{i j}^{-} \bar{N}_{j}\right), \quad 1 \leq i \leq n,
\end{array}\right.
$$

where for $-1<\alpha<1$, the function $\hat{r}(\alpha)$ is defined in Muroya [5, Lemma 2.2] (see Lemmas 2.6 and 2.7). Then for a sufficiently large $p \geq 0$, solutions 
of system (1.1) hold the contractivity.

Moreover, for a sufficiently large $p \geq 0$, solutions of system (1.1) hold the strong contractivity and the positive equilibrium $\boldsymbol{N}^{*}=\left(N_{1}^{*}, N_{2}^{*}, \ldots, N_{n}^{*}\right)^{T}$ of system (1.1) is globally asymptotically stable.

Since

$$
\lim _{\alpha \rightarrow-1+0} \hat{r}(\alpha)=0, \hat{r}(0)=2
$$

and $\hat{r}(\alpha)$ is a strictly monotone increasing function on $(-1,0]$ (see Muroya [5, Lemma 2.2]), by

$$
-1<-\frac{\sum_{j=1}^{n} \hat{\tilde{b}}_{i j}}{b_{i}} \leq 0, \quad 1 \leq i \leq n,
$$

there are cases that

$$
1<\hat{r}\left(-\frac{\sum_{j=1}^{n} \hat{\tilde{b}}_{i j}}{b_{i}}\right) \leq 2
$$

(see Uesugi et al. [11, Fig. 1]), which imply that there are cases such that the condition (1.15) improve the condition (1.12) (see Example 2.2).

By using a Lyapunov-like discrete function, Muroya [6, 8] obtained sufficient conditions that system (1.1) is globally asymptotically stable. But these conditions are different from the condition (1.15) (see Muroya [8, Corollary 2.2 and Remark 2.4]).

The organization of this paper is as follows. In Section 2, we study conditions of contractivity and give proofs of Theorems 1.1-1.3. Moreover, applying the same technique in Chen and Zhou [2] to (1.1), we obtain another contractivity (see Theorem 2.1). Examples are also given (see Examples 2.1-2.3).

\section{Conditions of contractivity}

We get a basic lemma (see Muroya [5, Lemma 3.1]).

Lemma 2.1 For two solutions $\left\{N_{i}(p)\right\}_{i=1}^{n}$ and $\left\{N_{i}^{*}(p)\right\}_{i=1}^{n}$ of system (1.1), we have 


$$
=\left\{\begin{array}{c}
N_{i}(p+1)-N_{i}^{*}(p+1) \\
\left.\times\left(N_{i}(p)-N_{i}^{*}(p)\right)-N_{i}(p) \frac{\exp \left(r_{i}(p)\left(t_{i}(p)-t_{i}^{*}(p)\right)\right)-1}{t_{i}(p)-t_{i}^{*}(p)}\right) \\
\left.\times \sum_{j=1}^{n} \sum_{l=0}^{m} b_{i j}^{l}(p)\left(N_{j}(p-l)-N_{j}^{*}(p-l)\right)\right\}, \\
\exp \left(r_{i}(p) t_{i}^{*}(p)\right)\left\{( 1 - b _ { i } ( p ) N _ { i } ( p ) N _ { i } ( p ) r _ { i } ( p ) ) \left(t_{i}(p) \neq 0,\right.\right. \\
-N_{i}(p) r_{i}(p) \sum_{j=1}^{n} \sum_{l=0}^{m} b_{i j}^{l}(p)\left(N_{j}(p-l)-N_{i}^{*}(p)\right) \\
\text { if } t_{i}(p)-t_{i}^{*}(p)=0,
\end{array}\right.
$$

where $t_{i}(p)$ and $t_{i}^{*}(p)$ are defined by (1.7).

Proof. From Eqs. (1.1) and (1.7), we have

$$
\left\{\begin{array}{l}
N_{i}(p+1)=N_{i}(p) \exp \left(r_{i}(p) t_{i}(p)\right), \\
N_{i}^{*}(p+1)=N_{i}^{*}(p) \exp \left(r_{i}(p) t_{i}^{*}(p)\right) .
\end{array}\right.
$$

Then, for $t_{i}(p)-t_{i}^{*}(p) \neq 0$,

$$
\begin{aligned}
& N_{i}(p+1)-N_{i}^{*}(p+1) \\
& =\exp \left(r_{i}(p) t_{i}^{*}(p)\right)\left\{\left(N_{i}(p)-N_{i}^{*}(p)\right)\right. \\
& \left.\quad+N_{i}(p) \frac{\exp \left(r_{i}(p)\left(t_{i}(p)-t_{i}^{*}(p)\right)\right)-1}{t_{i}(p)-t_{i}^{*}(p)}\left(t_{i}(p)-t_{i}^{*}(p)\right)\right\} \\
& =\exp \left(r_{i}(p) t_{i}^{*}(p)\right)\left\{\left(1-N_{i}(p) \frac{\exp \left(r_{i}(p)\left(t_{i}(p)-t_{i}^{*}(p)\right)\right)-1}{t_{i}(p)-t_{i}^{*}(p)} b_{i}(p)\right)\right. \\
& \quad \times\left(N_{i}(p)-N_{i}^{*}(p)\right)-N_{i}(p) \frac{\exp \left(r_{i}(p)\left(t_{i}(p)-t_{i}^{*}(p)\right)\right)-1}{t_{i}(p)-t_{i}^{*}(p)} \\
& \left.\quad \times \sum_{j=1}^{n} \sum_{l=0}^{m} b_{i j}^{l}(p)\left(N_{j}(p-l)-N_{j}^{*}(p-l)\right)\right\} .
\end{aligned}
$$

Similarly, for $t_{i}(p)-t_{i}^{*}(p)=0$, we have Eq. (2.1). 
Applying Lemma 2.1, we obtain the following basic result on the contractivity for solutions of system (1.1).

Lemma 2.2 If for any solution $\left\{N_{i}(p)\right\}_{i=1}^{n}$, there exists a solution $\left\{N_{i}^{*}(p)\right\}_{i=1}^{n}$, a positive constant $k \leq 1$ and a nonnegative integer $p_{0}$ such that for $p \geq p_{0}$, and $1 \leq i \leq n$,

$$
\left\{\begin{array}{c}
\left(b_{i}(p)-\sum_{j=1}^{n} \hat{\tilde{b}}_{i j}(p)\right) k_{i}(p) \geq 1-k \exp \left(-r_{i}(p) t_{i}^{*}(p)\right), \\
\text { if } b_{i}(p) k_{i}(p) \leq 1, \\
\left(b_{i}(p)+\sum_{j=1}^{n} \hat{\tilde{b}}_{i j}(p)\right) k_{i}(p) \leq k \exp \left(-r_{i}(p) t_{i}^{*}(p)\right)+1, \\
\text { if } b_{i}(p) k_{i}(p) \geq 1,
\end{array}\right.
$$

then solutions of system (1.1) hold the contractivity to the solution $\left\{N_{i}^{*}(p)\right\}_{i=1}^{n}$ for $p \geq p_{0}$.

Moreover, if $k<1$, then for $p \geq p_{0}$, solutions of system (1.1) hold the strong contractivity and

$$
\lim _{p \rightarrow \infty}\left(N_{i}(p)-N_{i}^{*}(p)\right)=0, \quad 1 \leq i \leq n .
$$

Proof. By (2.1) and (2.2), we have that for any solution $\left\{N_{i}(p)\right\}_{i=1}^{n}$ and $p \geq p_{0}$,

$$
\begin{aligned}
& \quad\left|N_{i}(p+1)-N_{i}^{*}(p+1)\right| \\
& \leq \exp \left(r_{i}(p) t_{i}^{*}(p)\right)\left(\left|1-b_{i}(p) k_{i}(p)\right|+\sum_{j=1}^{n} \hat{\tilde{b}}_{i j}(p) k_{i}(p)\right) \\
& \quad \times \max \left\{\left|N_{j}(p-l)-N_{j}^{*}(p-l)\right| \mid 1 \leq j \leq n, 0 \leq l \leq m\right\} \\
& \quad \leq k \max \left\{\left|N_{j}(p-l)-N_{j}^{*}(p-l)\right| \mid 1 \leq j \leq n, 0 \leq l \leq m\right\},
\end{aligned}
$$

from which we obtain the conclusion.

For the permanence of the system (1.1)-(1.2), Theorem 2.1 in Muroya [8] offer upper bounds $\bar{N}_{i}$ of $\lim \sup _{p \rightarrow \infty} N_{i}(p), 1 \leq i \leq n$ and lower bounds $\underline{N}_{i}>0$ of $\liminf \inf _{p \rightarrow \infty} N_{i}(p), 1 \leq i \leq n$.

Lemma 2.3 (See Muroya [8, Theorem 2.1]) Under the condition

$$
\left(\tilde{A}_{L}+\tilde{B}_{L}^{-}\right)^{-1} \boldsymbol{c}_{M}>\mathbf{0},
$$


for any solutions $\left\{N_{i}(p)\right\}_{i=1}^{n}$ of the system (1.1)-(1.2), it holds that

$$
\limsup _{p \rightarrow \infty} N_{i}(p) \leq \bar{N}_{i}, \quad 1 \leq i \leq n,
$$

where

$$
\left\{\begin{array}{l}
\text { for } i=1,2, \ldots, n \\
\bar{c}_{i M}=c_{i M}-\sum_{j=1}^{i-1} \tilde{b}_{i j L}^{-} \bar{N}_{j}, \\
\bar{N}_{i}= \begin{cases}\bar{c}_{i M} / b_{i L}, & \bar{r}_{i} \bar{c}_{i M} \leq 1, \\
e^{\bar{r}_{i} \bar{c}_{i M}-1} /\left(\bar{r}_{i} b_{i L}\right), & \bar{r}_{i} \bar{c}_{i M}>1,\end{cases} \\
\text { and } \boldsymbol{c}_{M}=\left[c_{1 M}, c_{2 M}, \ldots, c_{n M}\right]^{T} .
\end{array}\right.
$$

Moreover, if

$$
c_{i L}-\sum_{j=1}^{i-1} b_{i j M}^{-} \underline{N}_{j}-\sum_{j \neq i} b_{i j M}^{+} \bar{N}_{j}>0, \quad 1 \leq i \leq n,
$$

then

$$
\liminf _{p \rightarrow \infty} N_{i}(p) \geq \underline{N}_{i}, \quad 1 \leq i \leq n,
$$

where

$$
\left\{\begin{aligned}
\tilde{b}_{i M}= & b_{i M}+\sum_{l=1}^{m} b_{i i M}^{l} \\
& \times \exp \left\{-k_{l} \bar{r}_{i}\left(c_{i L}-b_{i M} \bar{N}_{i}-\sum_{j=1}^{i-1} b_{i j M}^{-} \underline{N}_{j}-\sum_{j=1}^{n} b_{i j M}^{+} \bar{N}_{j}\right)\right\} \\
\underline{\tilde{N}}_{i}= & \left(c_{i L}-\sum_{j=1}^{i-1} b_{i j M}^{-} \underline{N}_{j}-\sum_{j \neq i} b_{i j M}^{+} \bar{N}_{j}\right) / \tilde{b}_{i M} \\
\underline{N}_{i}= & \min \left(\underline{\tilde{N}}_{i}, \bar{N}_{i} \exp \left\{\overline { r } _ { i } \left(c_{i L}-\sum_{j=1}^{i-1} b_{i j M}^{-} \underline{N}_{j}\right.\right.\right. \\
& \left.\left.\left.-\sum_{j \neq i} b_{i j M}^{+} \bar{N}_{j}-\tilde{b}_{i M} \bar{N}_{i}\right)\right\}\right)>0, \quad 1 \leq i \leq n
\end{aligned}\right.
$$

Note that if (1.8) holds, then

$$
\bar{r}_{i} \bar{c}_{i M} \leq \bar{r} b_{i M} \bar{N}_{i} \leq 1, \quad 1 \leq i \leq n,
$$


and $\bar{N}_{i}=\tilde{N}_{i}, 1 \leq i \leq n$, where $\tilde{N}_{i}, i=1,2, \ldots, n$ are defined by

$$
\bar{c}_{i M}=c_{i M}-\sum_{j=1}^{i-1} \tilde{b}_{i j L}^{-} \bar{N}_{j}, \bar{N}_{i}=\frac{\bar{c}_{i M}}{b_{i L}}, \quad i=1,2, \ldots, n,
$$

that is, for $n$-dimensional vectors $\tilde{\boldsymbol{N}}=\left[\tilde{N}_{i}\right]$ and $\boldsymbol{c}_{M}=\left[c_{i M}\right]$,

$$
\tilde{\boldsymbol{N}}=\left(\tilde{A}_{L}+\tilde{B}_{L}^{-}\right)^{-1} \boldsymbol{c}_{M} .
$$

Now, we have the following lemma (cf. the proof of Wang and $\mathrm{Lu}[12$, Theorem 2] and Wang et al. [13]).

Lemma 2.4 Assume (2.3) and (2.6) and suppose that there exists a solution $\left\{N_{i}^{*}(p)\right\}_{i=1}^{n}$ of (1.1) such that (1.8) holds. Then, it holds that for any solution $\left\{N_{i}(p)\right\}_{i=1}^{n}$ of (1.1) and a sufficiently large $p \geq 0$,

$$
b_{i}(p) k_{i}(p) \leq 1, \quad 1 \leq i \leq n .
$$

Proof. Consider the function

$$
g(x)= \begin{cases}\left(e^{x}-1\right) / x, & x \neq 0 \\ 1, & x=0 .\end{cases}
$$

Then,

$$
g^{\prime}(x)= \begin{cases}\left(1 / x^{2}\right)\left\{(x-1) e^{x}+1\right\}, & x \neq 0, \\ 1 / 2, & x=0,\end{cases}
$$

and for

$$
h(x)=(x-1) e^{x}+1, \quad h^{\prime}(x)=x e^{x} .
$$

Then, $h(x) \geq h(0)=0, g^{\prime}(x) \geq 0$ and hence, $g(x)$ is positive and a strictly monotone increasing function of $x$ on $(-\infty,+\infty)$.

On the other hand, put $\bar{t}_{i}(p)=1-r_{i}(p) b_{i}(p) N_{i}(p)$. Then by Eqs. (1.7) and (1.8), for a sufficiently large $p \geq 0$,

$$
\begin{array}{r}
1 \geq \bar{t}_{i}(p) \geq 1-\bar{r}_{i} b_{i M} \bar{N}_{i} \geq 0, r_{i}(p)\left(c_{i}(p)-\sum_{j=1}^{i-1} \tilde{b}_{i j}^{-}(p) \bar{N}_{j}-t_{i}^{*}(p)\right) \leq 1, \\
1 \leq i \leq n,
\end{array}
$$

and 


$$
\begin{aligned}
r_{i}(p)\left(t_{i}(p)-t_{i}^{*}(p)\right)-\bar{t}_{i}(p) \leq & \left\{r_{i}(p)\left(c_{i}(p)-\sum_{j=1}^{i-1} \tilde{b}_{i j}^{-}(p) \bar{N}_{j}-t_{i}^{*}(p)\right)-1\right\} \\
& -r_{i}(p) \sum_{j=1}^{i-1} \sum_{l=0}^{m} b_{i j}^{l-}(p)\left(N_{j}(p-l)-\bar{N}_{j}\right)
\end{aligned}
$$

$\leq 0$

Thus,

$$
\begin{aligned}
b_{i}(p) k_{i}(p)= & r_{i}(p) b_{i}(p) N_{i}(p) g\left(r_{i}(p)\left(t_{i}(p)-t_{i}^{*}(p)\right)\right) \\
= & \left(1-\bar{t}_{i}(p)\right) g\left(\bar{t}_{i}(p)\right) \\
& +\left(1-\bar{t}_{i}(p)\right)\left\{g\left(r_{i}(p)\left(t_{i}(p)-t_{i}^{*}(p)\right)\right)-g\left(\bar{t}_{i}(p)\right)\right\} \\
\leq & \left(1-\bar{t}_{i}(p)\right) g\left(\bar{t}_{i}(p)\right) .
\end{aligned}
$$

Now, consider the following function

$$
p(x)=(1-x) \frac{e^{x}-1}{x}, \quad \text { for } \quad 0<x \leq 1 .
$$

Because $p^{\prime}(x)=\left(1 / x^{2}\right)\left\{1-\left(x^{2}-x+1\right) e^{x}\right\}$ and for $q(x)=\left(x^{2}-x+1\right) e^{x}$, $q^{\prime}(x)=x(x+1) e^{x}$, we have that $\sup _{0<x<1} p(x)=1$. Thus, we obtain that for a sufficiently large $p \geq p_{0},(2.12)$ holds. Hence, we complete the proof.

On the contractivity for solutions of system (1.1), we offer the following sufficient conditions for (2.2) in Lemma 2.2.

Lemma 2.5 In addition to the conditions in Lemma 2.4, assume that there exists a solution $\left\{N_{i}^{*}(p)\right\}_{i=1}^{n}$ such that for any solution $\left\{N_{i}(p)\right\}_{i=1}^{n}$ of (1.1), (1.8) and (1.10) hold. Then, for a sufficiently large $p \geq 0$, solutions of system (1.1) hold the contractivity to the solution $\left\{N_{i}^{*}(p)\right\}_{i=1}^{n}$. Moreover, if $k<1$, then for $p \geq p_{0}$, solutions of system (1.1) hold the strong contractivity and $\lim _{p \rightarrow \infty}\left(N_{i}(p)-N_{i}^{*}(p)\right)=0,1 \leq i \leq n$.

Proof. Since by (1.10), $\tilde{A}_{L}-\hat{\tilde{B}}_{M}$ is an $M$ matrix, we may assume, without loss of generality, that $\tilde{A}_{L}-\hat{\tilde{B}}_{M}$ is diagonally dominant matrix. Therefore, we may assume that for any $p \geq 0$,

$$
b_{i}(p)>\sum_{j=1}^{n} \hat{\tilde{b}}_{i j}(p), \quad 1 \leq i \leq n .
$$


By Lemma 2.4, $b_{i}(p) k_{i}(p) \leq 1$. If $t_{i}^{*}(p) \leq 0$, then $1-\exp \left(-r_{i}(p) t_{i}^{*}(p)\right) \leq 0$. Thus, by (2.13), the first equation of (2.2) holds for some constant $k \leq 1$. Suppose that $t_{i}^{*}(p)>0$. Since

$$
k_{i}(p)=N_{i}(p) \tilde{f}\left(t_{i}(p)-t_{i}^{*}(p) ; r_{i}(p)\right) \geq \underline{N}_{i} \tilde{f}\left(\bar{q}_{i} ; \underline{r}_{i}\right),
$$

by (1.8), we have

$$
\begin{aligned}
\left(b_{i}(p)-\sum_{j=1}^{n} \hat{\tilde{b}}_{i j}(p)\right) k_{i}(p) & \geq\left(b_{i}(p)-\sum_{j=1}^{n} \hat{\tilde{b}}_{i j}(p)\right) \underline{N}_{i} \tilde{f}\left(\bar{q}_{i} ; \underline{r}_{i}\right) \\
& \geq 1-k \exp \left(-\bar{r}_{i} t_{i}^{*}(p)\right) .
\end{aligned}
$$

Thus, the first equation of (2.2) holds and hence, by Lemmas 2.2 and 2.4, we obtain the conclusion.

From Lemmas 2.4 and 2.5, we establish sufficient conditions that solutions of (1.1) have the contractivity and the positive equilibrium $\boldsymbol{N}^{*}=$ $\left(N_{1}^{*}, N_{2}^{*}, \ldots, N_{n}^{*}\right)^{T}$ of (1.1) is globally asymptotically stable.

Proof of Theorem 1.1. By Lemmas 2.4 and 2.5, we get the conclusion.

An example of nonautonomous case in (1.1) which satisfies condition (2.2), is given in Example 2.1.

Proof of Theorem 1.2. We take $N_{i}^{*}(p)=N_{i}^{*}, 1 \leq i \leq n$. Then, each $t_{i}^{*}(p)=0$. Since we may assume $b_{i L}>\sum_{j=1}^{n} \tilde{\tilde{b}}_{i j}$ and $q_{i}>-\infty, 1 \leq i \leq n$, there exists a positive constant $k<1$ such that

$$
\left\{\begin{array}{l}
\underline{N}_{i} \tilde{f}\left(q_{i} ; \underline{r}_{i}\right) \geq(1-k) /\left(b_{i}(p)-\sum_{j=1}^{n} \hat{\tilde{b}}_{i j}(p)\right), \\
q_{i}=\min \left\{c_{i L}-b_{i M} \bar{N}_{i}-\sum_{j=1}^{n} b_{i j}^{+} \bar{N}_{j}, 0\right\} .
\end{array}\right.
$$

Hence, (1.8) becomes (1.12), and by Theorem 1.1, we obtain the conclusion.

Theorem 1.2 is an extension of Muroya [7, Theorem 1.1] for $n=1$ to $n \geq 1$.

For preparations to prove Theorem 1.3, we provide more three lemmas (see Muroya [5, Lemmas 2.1, 2.2 and 2.7] and their proofs). 
Lemma 2.6 Under the conditions that $0<\hat{Y}<\alpha$ for $0<\alpha<1$, and $\alpha<\hat{Y}<0$ for $-1<\alpha<0$, there exists a unique solution $\hat{Y}=\hat{Y}(\alpha)$ of the following equation:

$$
\frac{1}{1-\hat{Y}^{2}}=g(\hat{Y} ; \alpha), \quad-1<\alpha<1,
$$

where

$$
g(Y ; \alpha)= \begin{cases}\frac{1}{2(\alpha+Y)} \ln \frac{(1+\alpha)(1+Y)}{(1-\alpha)(1-Y)}, & Y \neq-\alpha \\ \frac{1}{1-\alpha^{2}}, & Y=-\alpha .\end{cases}
$$

In particular, $\hat{Y}(0)=0$ and

$$
\lim _{\alpha \rightarrow 0} \hat{Y}(\alpha)=\hat{Y}(0) .
$$

Remark 2.1 Note that for $0<|\alpha|<1$, the equation $1 /\left(1-Y^{2}\right)=g(Y ; \alpha)$ has another solution $\hat{Y}=-\alpha$, but this solution does not satisfy the conditions $0<\hat{Y}<\alpha$ for $0<\alpha<1$, and $\alpha<\hat{Y}<0$ for $-1<\alpha<0$.

Lemma 2.7 For $-1<\alpha<1$, let $\hat{Y}(\alpha)$ be defined in Lemma 2.6 and put

$$
\hat{r}(\alpha)=\frac{2(1+\alpha)}{1-\hat{Y}^{2}(\alpha)} \quad \text { and } \quad \hat{t}(\alpha)=\frac{\alpha+\hat{Y}(\alpha)}{1+\alpha} .
$$

Then, $\hat{r}(\alpha)$ is a strictly monotone increasing function of $\alpha$ on the interval $(-1,1)$, and

$$
\lim _{\alpha \rightarrow-1+0} \hat{r}(\alpha)=0 \quad \text { and } \quad \lim _{\alpha \rightarrow 1-0} \hat{r}(\alpha)=+\infty,
$$

and hence,

$$
\lim _{\alpha \rightarrow-1+0} \hat{Y}(\alpha)=-1 \quad \text { and } \quad \lim _{\alpha \rightarrow 1-0} \hat{Y}(\alpha)=1 .
$$

Moreover,

$$
\left\{\begin{aligned}
\hat{t}(\alpha)<1, f^{\prime}(\hat{t}(\alpha) ; \hat{r}(\alpha))=0, & \text { and } \\
f^{\prime}(t ; \hat{r}(\alpha))>0, \text { for }-\infty<t<\hat{t}(\alpha) \quad \text { for } \hat{t}(\alpha)<t<1 . & f^{\prime}(t ; \hat{r}(\alpha))<0
\end{aligned}\right.
$$


Hence, for any $0<r \leq \hat{r}(\alpha)$, we have

$$
\left\{\begin{array}{l}
f(t ; r) \leq f(t ; \hat{r}(\alpha)) \leq f(\hat{t}(\alpha) ; \hat{r}(\alpha))=2 /(1-\alpha), \text { for } t<1, \\
f(t ; \hat{r}(\alpha))<2 /(1-\alpha), \text { for } t<1 \text { and } t \neq \hat{t}(\alpha) .
\end{array}\right.
$$

Note that for $-1<\alpha<1, \hat{r}(\alpha)$ is defined by (1.14).

Lemma 2.8 For $\beta \gamma>0$, put

$$
\tilde{\tilde{f}}(x ; r, \beta, \gamma)=x \frac{e^{r(\beta-\gamma x)}-1}{\beta-\gamma x} .
$$

Then, for $t=1-(\gamma / \beta) x$ and $\tilde{r}=\beta r$, we have

$$
\tilde{\tilde{f}}(x ; r, \beta, \gamma)=\frac{1}{\gamma} f(t ; \tilde{r}) .
$$

Proof of Theorem 1.3. Put

$$
r=r_{i}(p), \beta=c_{i}-\sum_{j=1}^{i-1} \tilde{b}_{i j}^{-} \bar{N}_{j}, \gamma=b_{i} \quad \text { and } \quad \tilde{r}=\beta r .
$$

Then, by (1.15),

$$
\tilde{r} \leq \hat{r}\left(-\left(\sum_{j=1}^{n} \hat{\tilde{b}}_{i j}\right) / \gamma\right), \quad \text { and } \quad \hat{r}^{-1}(\tilde{r}) \leq-\left(\sum_{j=1}^{n} \hat{\tilde{b}}_{i j}\right) / \gamma .
$$

Since

$$
t_{i}(p) \leq\left(c_{i}-\sum_{j=1}^{i-1} \tilde{b}_{i j}^{-} \bar{N}_{j}\right)-b_{i} N_{i}(p)=\beta-\gamma N_{i}(p)
$$

and by Lemma 2.7,

$$
f(t, \tilde{r}) \leq \frac{2}{1-\hat{r}^{-1}(\tilde{r})}, \quad \text { for any } \quad t<1,
$$

by Lemma 2.8 , we have that

$$
\begin{aligned}
k_{i}(p) & =N_{i}(p) \tilde{f}\left(t_{i}(p) ; r_{i}(p)\right) \leq \tilde{\tilde{f}}\left(N_{i}(p) ; r, \beta, \gamma\right) \\
& =\frac{1}{\gamma} f\left(1-\frac{\gamma}{\beta} N_{i}(p) ; \tilde{r}\right) \leq \frac{1}{\gamma} \frac{2}{1-\hat{r}^{-1}(\tilde{r})} \\
& \leq 2 /\left(b_{i}+\sum_{j=1}^{n} \hat{\tilde{b}}_{i j}\right) .
\end{aligned}
$$


Thus, for $t_{i}^{*}(p)=0$ and $k=1$, we obtain the second part of (2.2) in Lemma 2.2. Moreover, if (1.15) holds, then by the assumptions, there is a positive constant $k=1$ such that for $p \geq p_{0},\left|1-b_{i} k_{i}(p)\right|+\left(\sum_{j=1}^{n} \hat{\tilde{b}}_{i j}\right) k_{i}(p) \leq k$. Then, by (2.2),

$$
\begin{array}{r}
\left|N_{i}(p+1)-N_{i}^{*}\right| \leq k \max \left\{\left|N_{j}(p-l)-N_{j}^{*}\right| \mid 1 \leq j \leq n, 0 \leq l \leq m\right\}, \\
1 \leq i \leq n .
\end{array}
$$

The remained parts of theorem are similar to the proof of Theorem 3.5 in Muroya [5]. Hence, we get the conclusion.

Now, we give another contractivity condition which is an extended result of Chen and Zhou [2]. Let

$$
N_{i}(p)=N_{i}^{*}(p) \exp \left(x_{i}(p)\right), \quad 1 \leq i \leq n .
$$

then, Eq. (1.1) is equivalent to

$$
\begin{aligned}
x_{i}(p+1)=x_{i}(p)-b_{i}(p) & N_{i}^{*}(p) f\left(x_{i}(p)\right) \\
& -\sum_{j=1}^{n} \sum_{l=0}^{m} b_{i j}^{l}(p) N_{j}^{*}(p) f\left(x_{j}(p-l)\right),
\end{aligned}
$$

where

$$
f(x)=e^{x}-1
$$

Therefore,

$$
\begin{aligned}
& x_{i}(p+1)=\left(1-b_{i}(p) N_{i}^{*}(p) \exp \left(\theta_{i}(p) x_{i}(p)\right)\right) x_{i}(p) \\
&-\sum_{j=1}^{n} \sum_{l=0}^{m} b_{i j}^{l}(p) N_{j}^{*}(p) \\
&\left.\quad \times \exp \left(\theta_{j}(p-l) x_{j}(p-l)\right)\right) x_{j}(p-l),
\end{aligned}
$$

where $\theta_{i}(p) \in[0,1], 1 \leq i \leq n$. Thus, similar to the proof of Theorem 3 in Chen and Zhou [2], we obtain the following theorem.

Theorem 2.1 Assume that (2.3) and (2.6) and suppose that there exist a solution $\left\{N_{i}^{*}(p)\right\}_{i=1}^{n}$ of (1.1) and a constant $\lambda$ such that

$$
\left|1-b_{i}(p) N_{i}^{*}(p)\right|+\sum_{j=1}^{n} \sum_{l=0}^{m}\left|b_{i j}^{l}(p)\right| N_{j}^{*}(p) \leq \lambda<1,1 \leq i \leq n .
$$


Then, for any solution $\left\{N_{i}(p)\right\}_{i=1}^{n}$ of $(1.1)$, solutions $\left\{\ln \left(N_{i}(p) / N_{i}^{*}(p)\right)\right\}_{i=1}^{n}$ of (2.24) hold the strong contractivity to zero solution, that is,

$$
\begin{aligned}
\max _{1 \leq i \leq n}\left|\ln \frac{N_{i}(p+1)}{N_{i}^{*}(p+1)}\right| \\
\quad \leq \lambda \max \left\{\left|\ln \frac{N_{i}(p-l)}{N_{i}^{*}(p-l)}\right| \mid 1 \leq i \leq n, 0 \leq l \leq m\right\},
\end{aligned}
$$

and we have

$$
\lim _{p \rightarrow \infty}\left(N_{i}(p)-N_{i}^{*}(p)\right)=0, \quad 1 \leq i \leq n .
$$

Example 2.1 Consider

$$
N_{1}(p+1)=N_{1}(p) \exp \left\{r_{1}(p)\left(c_{1}(p)-b_{1}(p) N_{1}(p)\right)\right\},
$$

where

$$
\begin{aligned}
r_{1}(p)=r_{1}, c_{1}(p)=1, b_{1}(2 p)=\frac{15}{16}, b_{1}(2 p+1)= & \frac{17}{16} e^{-r_{1} / 16} \\
p & =0,1,2, \ldots
\end{aligned}
$$

Assume $0<r_{1} \leq 15 / 17$. Clearly,

$$
\bar{r}_{1}=\underline{r}_{1}=r_{1}, \bar{c}_{1 M}=1, b_{1 L}=\frac{15}{16}<b_{1 M}=\frac{17}{16} e^{-r_{1} / 16} .
$$

Let $N_{1}^{*}(0)=1$; then

$$
N_{1}^{*}(2 p)=1, N_{1}^{*}(2 p+1)=e^{r_{1} / 16}, \quad p=0,1,2, \ldots
$$

Then,

$$
\left\{\begin{array}{l}
\bar{c}_{1 M}=c_{1 M}=1>0, \\
\bar{N}_{1}=1 / b_{1 L}, \\
\bar{r}_{1}\left(1-\inf _{p \geq 0} t_{1}^{*}(p)\right) \leq \bar{r}_{1} b_{1 M} \bar{N}_{1}<1, \\
\underline{N}_{1}=\min \left(1 / b_{1 M}, \bar{N}_{1} \exp \left\{r_{1}\left(1-b_{1 M} \bar{N}_{1}\right)\right\}\right), \\
q_{1}=\min \left(1-b_{1 M} \bar{N}_{1}-\left(1-b_{1 L}\right), 0\right)=\min \left(b_{1 L}-b_{1 M} / b_{1 L}, 0\right) .
\end{array}\right.
$$

By elementary caluculations, we can easily prove that there is a positive constant $k<1$ such that 


$$
\underline{N}_{1} \tilde{f}\left(q_{1}, r_{1}\right) \geq\left\{1-k \exp \left(-\frac{r_{1}}{16}\right)\right\} / b_{1 L} .
$$

Therefore, (1.8) with $k<1$ and (1.10) hold. Hence, by Theorem 1.1, we conclude that $\lim _{p \rightarrow \infty}\left(N_{1}(p)-N_{1}^{*}(p)\right)=0$.

Example 2.2 If $n=1$ and $r_{1}(p)=1, p \geq 0$, then Eq. (1.1) become the following form:

$$
\begin{array}{r}
N_{1}(p+1)=N_{1}(p) \exp \left\{c_{1}-b_{1} N_{1}(p)-\sum_{l=1}^{m} b_{11}^{l} N_{1}(p-l)\right\}, \\
p \geq 0 .
\end{array}
$$

Assume $b_{11}^{l} \geq 0,1 \leq l \leq m, b_{1}>\sum_{l=1}^{m} b_{11}^{l} \geq 0$, and

$$
0<c_{1} \leq 1, \quad \text { or } \quad 0<c_{1}<\hat{r}\left(-\left(\sum_{l=1}^{m} b_{11}^{l}\right) / b_{1}\right)
$$

Then, $N_{1}^{*}=\left(c_{1}\right) /\left(b_{1}+\sum_{l=1}^{m} b_{11}^{l}\right)>0,(2.3)$ and (2.6) are satisfies. Thus, by Lemma 2.3, $\limsup _{p \rightarrow \infty} N_{1}(p) \leq c_{1} / b_{1}$, and since (1.12) and (1.13) in Theorem 1.2 or (1.15) in Theorem 1.3 are satisfied. Thus, by Theorems 1.2 or 1.3 , the positive equilibrium $N_{1}^{*}$ of Eq. (2.31) is globally asymptotically stable.

Note that since $\lim _{\alpha \rightarrow-1+0} \hat{r}(\alpha)=0, \hat{r}(0)=2$ and $\hat{r}(\alpha)$ is a strictly monotone increasing function on $(-1,0]$ (see Muroya [5, Lemma 2.2]), by $-1<$ $-\left(\sum_{l=1}^{m} b_{11}^{l}\right) / b_{1} \leq 0$, there are cases that $1<\hat{r}\left(-\left(\sum_{l=1}^{m} b_{11}^{l}\right) / b_{1}\right) \leq 2$, which imply that there are cases such that the condition $0<c_{1}<\hat{r}\left(-\left(\sum_{l=1}^{m} b_{11}^{l}\right) / b_{1}\right)$ improves the condition $0<c_{1} \leq 1$. For example, if $b_{11}^{l}=0,1 \leq l \leq m$, then the former condition becomes $0<c_{1}<\hat{r}(0)=2$.

For the periodic case that $m=0$ and $b_{1}=b_{1}(p)$ and $c_{1}=c_{1}(p)$ are positive and periodic with a common positive period $\omega$ in (2.31), using a similar contractivity condition, Zhou and Zou [14] obtained another sufficient condition that a periodic solution is globally asymptotically stable if

$$
\sup _{p \geq 0} c_{1}(p) \leq 1+\ln \left\{2 \frac{\inf _{p \geq 0}\left(c_{1}(p) / b_{1}(p)\right)}{\sup _{p \geq 0}\left(c_{1}(p) / b_{1}(p)\right)}\right\} \leq 1+\ln 2<2 .
$$

For the case that $m=0$ and $b_{1}$ and $c_{1}$ are constants, the condition of Zhou and Zou [14] becomes $b_{1}>0$ and $0<c_{1} \leq 1+\ln 2$. In Eq. (2.32), there are cases that $1+\ln 2<c_{1}<\hat{r}(0)=2$ (see Uesugi et al. [11, Fig. 1]). 
Example 2.3 If $n=2, r_{1}(p)=r_{2}(p)=1, p \geq 0$, and $m=0$, then Eq. (1.1) becomes the following form:

$$
\left\{\begin{array}{l}
N_{1}(p+1)=N_{1}(p) \exp \left\{c_{1}-b_{1} N_{1}(p)-b_{12}^{0} N_{2}(p)\right\}, \\
N_{2}(p+1)=N_{2}(p) \exp \left\{c_{2}-b_{21}^{0} N_{1}(p)-b_{2} N_{2}(p)\right\}, p \geq 0 .
\end{array}\right.
$$

Assume that

$$
\begin{aligned}
b_{1}, b_{2}>0, b_{12}^{0} \geq 0, & b_{2} c_{1}-b_{12}^{0} c_{2}>0, \\
& -b_{21}^{0} c_{1}+b_{1} c_{2}>0 \text { and } c_{1}, c_{2}>0 .
\end{aligned}
$$

Then, $b_{1} b_{2}-b_{12}^{0} b_{21}^{0}>0$ and

$$
N_{1}^{*}=\frac{b_{2} c_{1}-b_{12}^{0} c_{2}}{b_{1} b_{2}-b_{12}^{0} b_{21}^{0}}>0, N_{2}^{*}=\frac{-b_{21}^{0} c_{1}+b_{1} c_{2}}{b_{1} b_{2}-b_{12}^{0} b_{21}^{0}}>0,
$$

Eqs. (2.3) and (2.6) are satisfied. Hence, by Lemma 2.3, $\lim _{\sup } \rightarrow \infty N_{i}(p) \leq$ $\bar{N}_{i}$. Moreover, since $b_{1}, b_{2}>0, b_{1} b_{2}-b_{12}^{0} b_{21}^{0}>0$ and $r_{1}(p)=r_{2}(p)=1$ imply (1.12) and (1.13), by Theorem 1.2, the positive equilibrium $\boldsymbol{N}^{*}=$ $\left(N_{1}^{*}, N_{2}^{*}\right)^{T}$ is globally asymptotically stable.

Note that the necessary and sufficient conditions of permanence in Saito, Ma and Hara [10, Corollary 1.1], are equal to Eq. (2.35).

Note that if

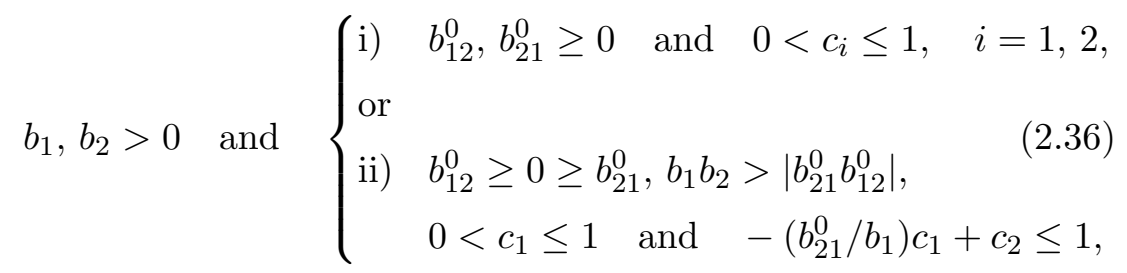

then Eqs. (2.34) and (2.35) are satisfied. For the system (2.33), the conditions of Theorem 2 in Wang and $\mathrm{Lu}$ [12], are equal to Eq. (2.34) and i) of Eq. (2.36), and the conditions of Corollary 4 in Wang and $\mathrm{Lu}$ [12], are equal to Eq. (2.34) and ii) of Eq. (2.36), and hence these satisfy just the above conditions Eqs. (2.34) and (2.36).

If

$$
\left\{\begin{array} { l } 
{ b _ { 1 } > | b _ { 1 2 } ^ { 0 } | , } \\
{ b _ { 2 } > | b _ { 2 1 } ^ { 0 } | , }
\end{array} \text { and } \quad \left\{\begin{array}{l}
c_{1}<\hat{r}\left(-\left|b_{12}^{0}\right| / b_{1}\right), \\
c_{2}-b_{21}^{0-} \bar{N}_{1}<\hat{r}\left(-\left|b_{21}^{0}\right| / b_{2}\right),
\end{array}\right.\right.
$$




$$
\text { where } \quad \bar{N}_{1}= \begin{cases}c_{1} / b_{1}, & c_{1} \leq 1, \\ e^{c_{1}-1} / b_{1}, & c_{1}>1,\end{cases}
$$

then Eqs. (1.14) is satisfied and hence, by Theorem 1.3, we have that the positive equilibrium $\boldsymbol{N}^{*}$ of Eq. (2.33) is globally asymptotically stable.

Examples 2.2 and 2.3 show us that for the autonomous case of system (1.1), Theorems 1.2 and 1.3 are some extended results of Muroya [5, 7], and Wang and $\mathrm{Lu}$ [12, Theorem 2 and Corollary 4], and Wang et al. [13] to the cases $n \geq 2$ and $m \geq 0$.

For the periodic case that $c_{i}=c_{i}(p), b_{i}=b_{i}(p)$ and $b_{i j}=b_{i j}(p), 1 \leq$ $i, j \leq 2$ are positive and periodic with a common positive period $\omega$ in Eq. (2.33), Chen and Zhou [2] obtained another sufficient condition (10) in Chen and Zhou [2, Theorem 3], which is an extension of the result of Zhou and Zou [14] to this system. For a solution $\boldsymbol{N}(p)=\left(N_{1}(p), N_{2}(p)\right)^{T}$ and a periodic solution $\boldsymbol{N}^{*}(p)=\left(N_{1}^{*}(p), N_{2}^{*}(p)\right)^{T}$ of this system, the condition (10) in Chen and Zhou [2] implies that for some constant $0 \leq \lambda<1$ and nonnegative integer $p_{0}$, it holds that for any $p \geq p_{0}$,

$$
\max _{1 \leq i \leq 2}\left|\ln \frac{N_{i}(p+1)}{N_{i}^{*}(p+1)}\right| \leq \lambda \max _{1 \leq i \leq 2}\left|\ln \frac{N_{i}(p)}{N_{i}^{*}(p)}\right|
$$

which denotes that for $p \geq p_{0}$, solutions $\left\{\ln \left(N_{i}(p) / N_{i}^{*}(p)\right)\right\}_{i=1}^{2}$ hold the strong contractivity to zero solution of $(2.24)$.

Acknowledgment This work was partially done during the stay of the author at the University of Trieste. The author wishes to express his gratitude to Professors Alfredo Bellen and Marino Zennaro for their hospitality during his visit. The author also wishes to thank to the anonymous referees for their valuable comments.

\section{References}

[1] Berman A. and Plemmons R.J., Nonnegative Matrices in Mathematical Sciences. Academic Press, New York, 1979.

[2] Chen Y. and Zhou Z., Stable periodic solution of a discrete periodic Lotka-Volterra competition system. J. Math. Anal. Appl. 277 (2003), 358-366.

[ 3 ] Hofbauer J., Hutson V. and Jansen W., Coexistence for systems governed by difference equations of Lotka-Volterra type. J. Math. Biol. 25 (1987), 553-570.

[4] Lu Z. and Wang W., Permanence and global attractivity for Lotka-Volterra difference systems. J. Math. Biol. 39 (1999), 269-282. 
[5] Muroya Y., Persistence, contractivity and global stability in logistic equations with piecewise constant delays. J. Math. Anal. Appl. 270 (2002), 602-635.

[6] Muroya Y., Persistence and global stability for discrete models of nonautonomous Lotka-Volterra type. J. Math. Anal. Appl. 273 (2002), 492-511.

[7] Muroya Y., A sufficient condition on global stability in a logistic equation with piecewise constant arguments. Hokkaido Math. J. 32 (2003), 75-83.

[8] Muroya Y., Global stability in discrete models of nonautonomous Lotka-Volterra type. Hokkaido Math. J. 33 (2004), 115-126.

[9] Saito Y., Hara T. and Ma W., Harmless delays for permanence and impersistence of Lotka-Volterra discrete predator-prey sysrem. Nonlinear Analysis 50 (2002), 703715.

[10] Saito Y., Ma W. and Hara T., A necessary and sufficient condition for permanence of a Lotka-Volterra discrete system with delays. J. Math. Anal. Appl. 256 (2001), $162-174$.

[11] Uesugi K. Muroya Y. and Ishiwata E., On the global attractivity for a logistic equation with piecewise constant arguments. J. Math. Anal. Appl. 294 (2004), 560-580.

[12] Wang W. and Lu Z., Global stability of discrete models of Lotka-Volterra type. Nonlinear Analysis 35 (1999), 1019-1030.

[13] Wang W., Mulone G., Salemi F. and Salone V., Global stability of discrete population models with time delays and fluctuating environment. J. Math. Anal. Appl. 264 (2001), 147-169.

[14] Zhou Z. and Zou X., Stable periodic solution in a discrete periodic logistic equation. Appl. Math. Lett. 16 (2003), 165-171.

Department of Mathematical Sciences Waseda University

3-4-1 Ohkubo Shinjuku-ku

Tokyo, 169-8555 Japan

E-mail: ymuroya@waseda.jp 